\title{
HIGHLIGHTS
}

GENETICS

\section{BRAF but not KRAS is prognostic in colon cancer}

Around $35-42 \%$ of colorectal cancers (CRC) harbor mutations in KRAS, which occur early in the course of the disease. Patients with metastatic CRC who have KRAS mutations derive no benefit from anti-EGFR antibodies, and the $B R A F$ V600E mutation also confers resistance to such therapies. Roth et al. prospectively

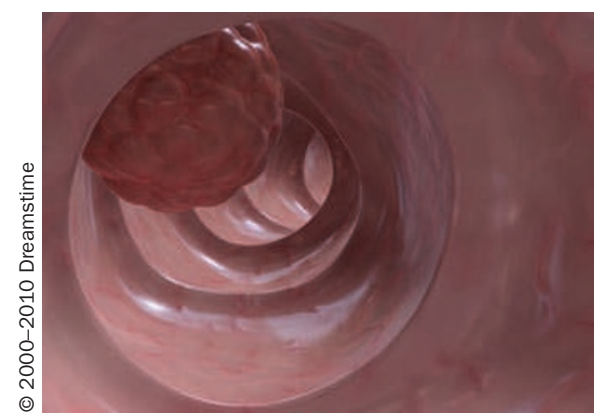

examined the prognostic role of $B R A F$ and $K R A S$ mutations in a large randomized study, and found that BRAF mutations are a strong prognostic factor for overall survival particularly in patients with stage II disease who have either low or no microsatellite instability.

DNA was collected from 1,404 patients with stage II or III adenocarcinoma of the colon. KRAS and BRAF V600E mutations were identified in $37 \%$ and $7.9 \%$ of samples, respectively. These mutation rates did not differ significantly between stage II and III tumors. Multivariate analysis revealed that KRAS mutation was significantly associated with tumor grade, and right-sided tumors. The BRAF V600E mutation was significantly associated with female sex, tumor grade, age over 60 years, high levels of MSI, and right-sided tumors.
In multivariate analysis, mutated KRAS did not have prognostic value in determining relapse-free survival or overall survival. The BRAF V600E mutation was not prognostic for relapsefree survival; however, it did predict an overall survival benefit, especially in patients with low levels of MSI or stable MSI tumors.

Roth and his team conclude KRAS has no prognostic value in CRC but their evidence suggests that $B R A F$ is prognostic for overall survival, despite both proteins functioning in the same pathway.

\section{Lisa Hutchinson}

Original article Roth, A. D. et al. Prognostic role of KRAS and BRAF in stage II and III resected colon cancer: results of the translational study on the PETACC-3, EORTC 40993, SAKK 60-00 trial. J. Clin. Oncol. 28, 466-474 (2010) 